\title{
NONLINEAR STABILITY ANALYSIS OF A SPINNING TOP WITH AN INTERIOR LIQUID-FILLED CAVITY
}

\author{
Giovanni P. Galdi ${ }^{1, *}$ And Giusy Mazzone ${ }^{2}$
}

\begin{abstract}
Consider the motion of the coupled system, $\mathcal{S}$, constituted by a (non-necessarily symmetric) top, $\mathcal{B}$, with an interior cavity, $\mathcal{C}$, filled up with a Navier-Stokes liquid, $\mathcal{L}$. A particular steady-state motion $\overline{\mathrm{s}}$ (say) of $\mathcal{S}$, is when $\mathcal{L}$ is at rest with respect to $\mathcal{B}$, and $\mathcal{S}$, as a whole rigid body, spins with a constant angular velocity $\overline{\boldsymbol{\omega}}$ around a vertical axis passing through its center of mass $G$ in its highest position (upright spinning top). We then provide a complete characterization of the nonlinear stability of $\bar{s}$ by showing, roughly speaking, that $\overline{\boldsymbol{s}}$ is stable if and only if $|\overline{\boldsymbol{\omega}}|$ is sufficiently large, all other physical parameters being fixed. Moreover we show that, unlike the case when $\mathcal{C}$ is empty, under the above stability conditions, the top will eventually return to the unperturbed upright configuration.
\end{abstract}

Mathematics Subject Classification. 35Q35, 35Q30, 35B35, 35B40, 75D05.

Received February 5, 2020. Accepted November 30, 2020.

\section{INTRODUCTION}

The stability of an upright spinning top is a renowned, classical problem in rigid body dynamics. More precisely, the top is a rigid body, $\mathcal{B}$, that moves while keeping one of its points, $O$, fixed at all times. Among the many motions that $\mathcal{B}$ can execute, particularly interesting is the one where $\mathcal{B}$ spins with constant angular velocity, $\overline{\boldsymbol{\omega}}$, around the vertical axis, a, passing through $O$ and its center of mass $G$ at its highest position, and coinciding with one of the principal axes of inertia $\boldsymbol{e}_{\mathcal{B}}$ (say). The above-mentioned problem consists exactly in studying the stability properties of such rotational motion. It is then well known that, if $|\overline{\boldsymbol{\omega}}|$ is "sufficiently large" and the principal moment of inertia around $\boldsymbol{e}_{\mathcal{B}}$ is a maximum, then the motion is stable and the perturbed motion will be a combined precession-nutation around a ([22], Ex. 9.7C).

In this paper we investigate the analogous question when the top has an interior cavity, $\mathcal{C}$, entirely filled with a viscous liquid, $\mathcal{L}$. In this case the basic motion is the same as above, with $\mathcal{L}$ at rest with respect to $\mathcal{B}$ and the coupled system $\mathcal{S}:=\mathcal{B} \cup \mathcal{L}$ rotating as a whole rigid body around the direction a coinciding with $\boldsymbol{e}_{\mathcal{S}}$. We denote by $\bar{s}$ this particular unperturbed steady-state. Mainly due to its important technological applications ([2] and the references therein), this problem has attracted the attention of a number of applied mathematicians, especially from the Russian School. As a matter of fact, Sobolev [28] was the first to give sufficient conditions for the stability of $\overline{\mathbf{s}}$, in the case when the liquid is inviscid (Euler), within the linearized approximation, namely, when all nonlinear terms in the relevant equations are entirely disregarded. Precisely, let $A, B$ and $C$ be the

Keywords and phrases: Fluid-solid interaction, Navier-Stokes equations, rigid body, stabilty.

1 Department of Mechanical Engineering and Materials Science, University of Pittsburgh, Pittsburgh, USA.

2 Department of Mathematics and Statistics, Queen's University, Kingston, Canada.

* Corresponding author: galdi@pitt.edu

(C) The authors. Published by EDP Sciences, 2021 
principal moments of inertia of $\mathcal{S}$ with respect to $O$ with $C$ moment of inertia about $\boldsymbol{e}_{\mathcal{S}}$. In [28] it is then shown that a sufficient condition for linear stability is that

$$
C>M:=\max \{A, B\}, \quad \bar{\omega}^{2}>\frac{\beta^{2}}{C-M},
$$

where $\beta^{2}$ is the material constant defined in (2.2). Successively, by a clever but formal ${ }^{1}$ application of the classical Liapunov method, Rumyantsev [25] proved that the requests in (1.1) in fact ensure full nonlinear stability when $\mathcal{L}$ is viscous (Navier-Stokes). Instability results were further furnished by Chernus'ko [1] and Smirnova $[26,27]$ by means of a formal asymptotic expansion obtained for small relevant Reynolds number, and suitable symmetry assumptions on the shape of the cavity. More recently, Kostyuchenko et al. [15] have shown, among other things, that, if $\mathcal{L}$ is a Navier-Stokes liquid, under the assumption $(1.1)_{1}$, the request in $(1.1)_{2}$ is, basically, necessary and sufficient for the stability of $\bar{s}$. More precisely, (1.1) ensures stability, whereas the conditions

$$
C>M:=\max \{A, B\}, \quad \bar{\omega}^{2}<\frac{\beta^{2}}{C-M},
$$

implies that $\bar{s}$ is unstable. However, again as in [28], the result in [15] is proved for the linearized set of equations only; see also $[14,31]$.

Over the last few years, the present authors and their associates have started a systematic and stringent analysis of the motion of a rigid body with an interior cavity entirely filled with a viscous liquid [4, 6-10, 17-20]. In particular, in Section 8 of [10], it has been shown in a rigorous way that condition (1.1) guarantees the full nonlinear stability of the steady-state $\bar{s}$ in a very large class of perturbations (weak solutions). However, the instability counterpart of this result requires a condition that is more restrictive than that stated in (1.2).

The objective of this paper is to furnish a rigorous, complete and detailed analysis of nonlinear asymptotic stability of the steady-state motion $\overline{\mathbf{s}}$ of $\mathcal{S}$. The method we employ is based on the study of the spectral properties of the relevant linear operator -obtained by linearizing the full nonlinear operator around $\bar{s}$ - in combination with a "generalized linearization principle" of the type introduced in [8]; see Theorem 3.2. One of the key ingredient of our proof is hypothesis (H5) on the nonlinear operator of our evolution equation (2.12). Such assumption ensures that the null set of the relevant linear operator is contained in the manifold of equilibria for (2.12), and it defines a stable center manifold which attracts nearby trajectories. We are thus able to show that the conditions stated in (1.1) ensure the nonlinear asymptotic stability of $\bar{s}$ in a suitable regularity class of perturbations (Thm. 7.1). Here, by "asymptotic stability" we mean that $\bar{s}$ is stable in the classical sense of Liapunov and, moreover, the generic perturbed motion with initial data in an appropriate neighborhood of $\overline{\mathbf{s}}$ will converge, exponentially fast, to a steady-state where the top continues to spin in the upright position. This "stabilizing effect" is just due to the presence the liquid since, as we noted earlier on, if the cavity is empty the perturbed motion of the top is precession-nutation-like around the vertical axis through the fixed point $O$. In addition, we provide two sufficient conditions for the nonlinear instability of $\bar{s}$. Precisely, we show that $\bar{s}$ is unstable if either (1.2) holds or else the principal moment of inertia $C$ is not a maximum (Thm. 7.3). The latter result, in its full generality, is entirely new.

The plan of the paper is as follows. In Section 2 we furnish a mathematical formulation of the stability problem along with some preparatory results. In Section 3, Theorem 3.2, we prove a "generalized linearization principle" for the trivial solution of an abstract evolution problem in a Banach space. Unlike the classical "linearization principle" ([12], Thm. 5.1.1) where, for stability, the spectrum of the linearization, L (say), must have eigenvalues with positive real part, our theorem allows 0 to be in the spectrum of $\mathbf{L}$, on condition that it is a semi-simple eigenvalue. We also show that, if $\mathbf{L}$ has at least one eigenvalue with negative real part, then the trivial solution is unstable; see Theorem 3.3. This result would follow also from ([12], Thm. 5.1.5); however, we give an independent and simple proof for the sake of completeness. In Section 4, we show that the linearization

\footnotetext{
${ }^{1}$ No existence theory is provided for the underlying initial-boundary value problem.
} 


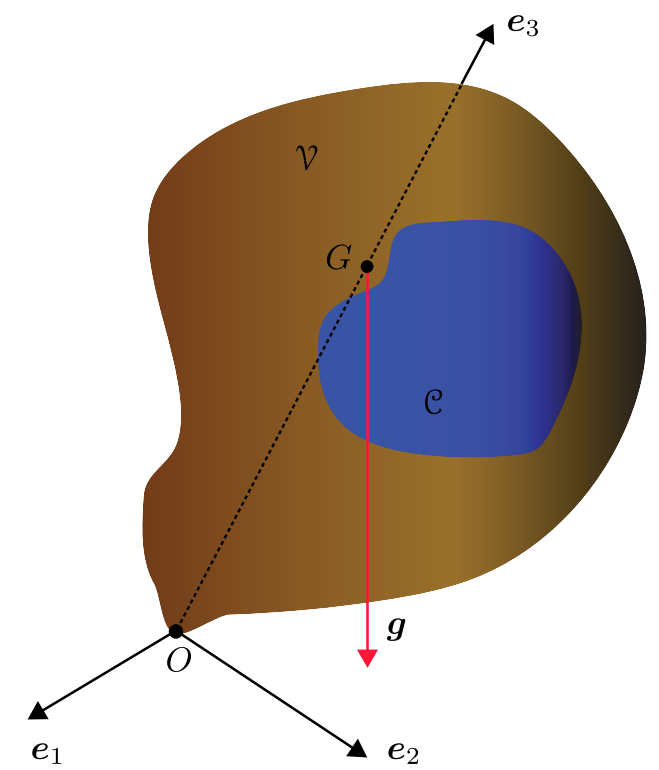

FiguRE 1. A liquid-filled spinning top.

around $\overline{\mathbf{s}}$ obeys all regularity and spectral properties of the abstract operator $\mathbf{L}$. The study of the location of the spectrum, $\sigma$, of the linearization is then carried in Section 5. In Section 6 we prove that the relevant nonlinear operator in our stability problem satisfies the assumptions of the analogous operator in the abstract setting. Finally, in Section 7 we are able to apply our abstract results to study the stability properties of $\overline{\mathbf{s}}$.

\section{FORMULATION OF THE PROBLEM AND PRELIMINARY CONSIDERATIONS}

In the following and for the rest of this paper, boldfaced lower-case letters will denote vector fields, whereas boldfaced capital letters will denote operators and tensor fields.

Let $\mathcal{S}:=\mathcal{B} \cup \mathcal{L}$ be the physical system constituted by a rigid body $\mathcal{B}$ possessing an interior cavity entirely filled with a Navier-Stokes liquid $\mathcal{L}$. We denote by $\mathcal{V}$ and $\mathcal{C}$ the spatial region occupied by $\mathcal{B}$ and $\mathcal{L}$, respectively. In mathematical terms, $\mathcal{V}$ and $\mathcal{C}$ are two bounded domains of $\mathbb{R}^{3}$ with $\overline{\mathcal{C}} \subset \mathcal{V}$. We suppose that $\mathcal{C}$ is of class $C^{2}$. We assume that $\mathcal{S}$ moves under the action of the gravity, $\boldsymbol{g}$, while keeping one of its points, $O$, fixed at all times with respect to an inertial frame (see Fig. 1). Denote by $\mathbb{I}$ the inertia tensor of $\mathcal{S}$ with respect to $O, \boldsymbol{e}_{1}, \boldsymbol{e}_{2}$, and $\boldsymbol{e}_{3}$ its (ortho-normalized) eigenvectors and $A, B$, and $C$ corresponding eigenvalues (principal moments of inertia). Moreover, let $\mathcal{F}:=\left\{O, \boldsymbol{e}_{1}, \boldsymbol{e}_{2}, \boldsymbol{e}_{3}\right\}$ denote the principal frame of inertia. We shall suppose that the center of mass, $G$, of $\mathcal{S}$ belongs to the axis $\overrightarrow{O e}_{3}$, and $G \neq O$. Also, we orient $\mathcal{F}$ in a way that the only non-zero coordinate, $\ell$, of $G$ in $\mathcal{F}$ is positive. Thus, the equations governing the motion of $\mathcal{S}$ in the body-fixed frame $\mathcal{F}$ are given by $[18,21]$

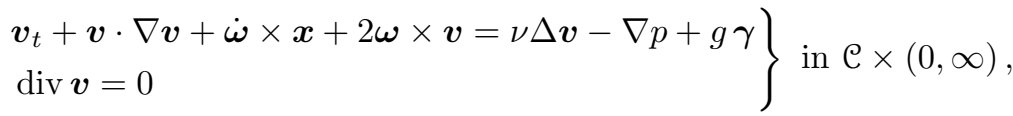

$$
\begin{aligned}
& \left.\begin{array}{l}
\mathbb{I} \cdot(\dot{\boldsymbol{\omega}}-\dot{\boldsymbol{a}})+\boldsymbol{\omega} \times \mathbb{I} \cdot(\boldsymbol{\omega}-\boldsymbol{a})=\beta^{2} \boldsymbol{e}_{3} \times \boldsymbol{\gamma} \\
\dot{\boldsymbol{\gamma}}+\boldsymbol{\omega} \times \boldsymbol{\gamma}=\mathbf{0}
\end{array}\right\} \text { in }(0, \infty), \\
& \boldsymbol{v}=\mathbf{0} \quad \text { on } \partial \mathcal{C} \times(0, \infty) \text {. }
\end{aligned}
$$


Here $\boldsymbol{v}, \rho, \mu \equiv \rho \nu$ and $\boldsymbol{\omega}$ denote, respectively, relative velocity, density, shear viscosity coefficient of $\mathcal{L}$ and angular velocity of $\mathcal{B}$. Moreover,

$$
\beta^{2}:=M \ell g, \quad \boldsymbol{a}:=-\rho \mathbb{I}^{-1} \cdot \int_{\mathcal{C}} \boldsymbol{x} \times \boldsymbol{v}, \quad p:=\frac{\gamma}{\rho} \mathrm{p}-\frac{\gamma}{2}(\boldsymbol{\omega} \times \boldsymbol{x})^{2},
$$

where $\mathrm{p}$ is the pressure field of $\mathcal{L}$, while $M$ is the total mass of $\mathcal{S}$, and $g=|\boldsymbol{g}|$. Finally, $\boldsymbol{\gamma}:=\boldsymbol{g} / g$ stands for the direction of the gravity, which, in the frame $\mathcal{F}$, is time-dependent. Notice that

$$
\gamma^{2}(t)=1, \text { for all } t \geq 0
$$

As it is immediately checked, (2.1) admits a class of time independent (steady-state) solutions $(\overline{\boldsymbol{v}}(\mathbf{x}), \overline{\boldsymbol{\omega}}, \bar{\gamma})$, characterized by the conditions

$$
\overline{\boldsymbol{v}} \equiv \mathbf{0}, \overline{\boldsymbol{\omega}}=\lambda \bar{\gamma}, \quad \bar{\gamma}=-\boldsymbol{e}_{3}, \quad \lambda \in \mathbb{R} \backslash\{0\} .
$$

From the physical viewpoint, such solutions describe those motions of $\mathcal{S}$, where the liquid is "frozen" in the cavity and $\mathcal{S}$ rotates as a whole rigid body around $\boldsymbol{e}_{3}$ with constant angular velocity $\boldsymbol{\omega}$, and its center of mass at its highest position ("upright spinning top").

The main objective of this paper is to characterize the stability properties of these motions.

To this end, let

$$
(\overline{\boldsymbol{v}}+\boldsymbol{v}, \overline{\boldsymbol{\omega}}+\boldsymbol{\omega}, \bar{\gamma}+\boldsymbol{z}),
$$

be a generic "perturbed motion" around (2.4). Then, in view of (2.1) the "perturbation" $(\boldsymbol{v}, \boldsymbol{\omega}, \boldsymbol{z})$ satisfies the following set of equations

$$
\left.\begin{array}{l}
\left.\begin{array}{l}
\boldsymbol{v}_{t}+\dot{\boldsymbol{\omega}} \times \boldsymbol{x}+\boldsymbol{v} \cdot \nabla \boldsymbol{v}+2\left(\boldsymbol{\omega}-\lambda \boldsymbol{e}_{3}\right) \times \boldsymbol{v}=\nu \Delta \boldsymbol{v}-\nabla p \\
\operatorname{div} \boldsymbol{v}=0
\end{array}\right\} \text { on } \mathcal{C} \times(0, \infty), \\
\mathbb{I} \cdot(\dot{\boldsymbol{\omega}}-\dot{\boldsymbol{a}})+\boldsymbol{\omega} \times \mathbb{I} \cdot(\boldsymbol{\omega}-\boldsymbol{a})-\lambda \boldsymbol{e}_{3} \times \mathbb{I} \cdot(\boldsymbol{\omega}-\boldsymbol{a})-\lambda C \boldsymbol{\omega} \times \boldsymbol{e}_{3}=\beta^{2} \boldsymbol{e}_{3} \times \boldsymbol{z} \\
\dot{\boldsymbol{z}}+\boldsymbol{\omega} \times \boldsymbol{z}-\boldsymbol{\omega} \times \boldsymbol{e}_{3}-\lambda \boldsymbol{e}_{3} \times \boldsymbol{z}=\mathbf{0} \\
\boldsymbol{v}=\mathbf{0} \quad \text { on } \partial \mathcal{C} \times(0, \infty) .
\end{array}\right\} \text { in }(0, \infty),
$$

Because of (2.3), the perturbation field $\boldsymbol{z}$ must satisfy the constraint

$$
\boldsymbol{z}(t) \cdot \boldsymbol{z}(t)-2 \boldsymbol{z}(t) \cdot \boldsymbol{e}_{3}=0, \text { all } t \geq 0
$$

However, dot-multiplying $(2.6)_{4}$ a first time by $\boldsymbol{z}$, a second time by $-\boldsymbol{e}_{3}$ and summing side by side the resulting equations, we find

$$
\frac{\mathrm{d}}{\mathrm{d} t}\left(\boldsymbol{z} \cdot \boldsymbol{z}-2 \boldsymbol{z} \cdot \boldsymbol{e}_{3}\right)=0, \text { for all } t \geq 0 .
$$

As a consequence, (2.7) is equivalent just to require that the initial data $\boldsymbol{z}(0)$ satisfies:

$$
\boldsymbol{z}(0) \cdot \boldsymbol{z}(0)-2 \boldsymbol{z}(0) \cdot \boldsymbol{e}_{3}=0 \text {. }
$$


Our next objective is to rewrite (2.6) as an evolution equation in an appropriate Hilbert space. To this end, let

$$
L_{\sigma}^{2}(\mathcal{C}):=\left\{\boldsymbol{v} \in L^{2}(\mathcal{C}): \operatorname{div} \boldsymbol{v}=0 \quad \text { in } \mathcal{C}, \boldsymbol{v} \cdot \boldsymbol{n}=0 \quad \text { on } \partial \mathfrak{C}\right\}
$$

and define the Hilbert space

$$
X:=\left\{\boldsymbol{u}=(\boldsymbol{v}, \boldsymbol{\omega}, \boldsymbol{z})^{T} \in L_{\sigma}^{2}(\mathcal{C}) \oplus \mathbb{R}^{3} \oplus \mathbb{R}^{3}\right\}
$$

endowed with the inner product

$$
\left\langle\boldsymbol{u}_{1}, \boldsymbol{u}_{2}\right\rangle=\int_{\mathcal{C}} \rho \boldsymbol{v}_{1} \cdot \boldsymbol{v}_{2} \mathrm{~d} V+\boldsymbol{\omega}_{1} \cdot \mathbb{I} \cdot \boldsymbol{\omega}_{2}+\boldsymbol{z}_{1} \cdot \boldsymbol{z}_{2}
$$

and associated norm

$$
\|\boldsymbol{u}\|:=\sqrt{\langle\boldsymbol{u}, \boldsymbol{u}\rangle}
$$

We then introduce the operators:

$$
\begin{aligned}
& \boldsymbol{I}: \boldsymbol{u} \in X \mapsto \boldsymbol{I} \boldsymbol{u}:=(\boldsymbol{v}+\mathrm{P}(\boldsymbol{\omega} \times \boldsymbol{x}), \mathbb{I} \cdot(\boldsymbol{\omega}-\boldsymbol{a}), \boldsymbol{z})^{\top} \in X, \\
& \boldsymbol{A}: \boldsymbol{u} \in \mathrm{D}(\boldsymbol{A}):=\left[W^{2,2}(\mathrm{C}) \cap W_{0}^{1,2}(\mathrm{C}) \cap L_{\sigma}^{2}(\mathrm{C})\right] \oplus \mathbb{R}^{3} \oplus \mathbb{R}^{3} \subset X \mapsto \boldsymbol{A u}:=(-\nu \mathrm{P} \Delta \boldsymbol{v}, \boldsymbol{\omega}, \boldsymbol{z})^{\top} \in X, \\
& \begin{aligned}
\boldsymbol{B}: \boldsymbol{u} \in X \mapsto \boldsymbol{B} \boldsymbol{u}:= & \left(2 \mathrm{P}\left(-\lambda \boldsymbol{e}_{3} \times \boldsymbol{v}\right),-\lambda \boldsymbol{e}_{3} \times \mathbb{I} \cdot \boldsymbol{\omega}-\lambda C \boldsymbol{\omega} \times \boldsymbol{e}_{3}+\lambda \boldsymbol{e}_{3} \times \mathbb{I} \cdot \boldsymbol{a}-\beta^{2} \boldsymbol{e}_{3} \times \boldsymbol{z}-\boldsymbol{\omega},\right. \\
& \left.\quad-\boldsymbol{\omega} \times \boldsymbol{e}_{3}-\lambda \boldsymbol{e}_{3} \times \boldsymbol{z}-\boldsymbol{z}\right)^{\top} \in X, \\
\boldsymbol{N}: \boldsymbol{u} \in \mathrm{D}(\boldsymbol{A}) \subset X \mapsto & \boldsymbol{N}(\boldsymbol{u}):=(-2 \mathrm{P}(\boldsymbol{\omega} \times \boldsymbol{v})-\mathrm{P}(\boldsymbol{v} \cdot \nabla \boldsymbol{v}),-\boldsymbol{\omega} \times \mathbb{I} \cdot(\boldsymbol{\omega}-\boldsymbol{a}),-\boldsymbol{\omega} \times \boldsymbol{z})^{\top} \in X,
\end{aligned}
\end{aligned}
$$

with P Helmholtz projection from $L^{2}(\mathcal{C})$ onto $L_{\sigma}^{2}(\mathcal{C})$.

From Section 6.2.3 of [14], we deduce the following.

Lemma 2.1. The operator $\boldsymbol{I}$ is bounded, positive with a bounded inverse.

In view of this lemma, we may set

$$
\mathbf{A}=\boldsymbol{I}^{-1} \boldsymbol{A}, \quad \mathbf{B}=\boldsymbol{I}^{-1} \boldsymbol{B}, \quad \mathbf{L}=\mathbf{A}+\mathbf{B}, \quad \mathbf{N}=\boldsymbol{I}^{-1} \boldsymbol{N},
$$

where $\mathrm{D}(\mathbf{L})=\mathrm{D}(\mathbf{N}) \equiv \mathrm{D}(\boldsymbol{A})$, and deduce that the system of equations (2.6) can be formally written as an evolution equation in the Hilbert space $X$ :

$$
\frac{\mathrm{d} \boldsymbol{u}}{\mathrm{d} t}+\mathbf{L} \boldsymbol{u}=\mathbf{N}(\boldsymbol{u})
$$

The stability of the steady-state solution (2.4) is then reduced to the stability of the solution $\boldsymbol{u}=\mathbf{0}$ to (2.12). The study of the latter will be performed as a result of the general stability theory - presented in next section that is founded upon suitable functional and spectral properties of the linear operator $\mathbf{L}$. 


\section{Stability PROPERTies FOR AN ABSTRACT EVOLUTION PROBLEM}

Objective of this section is to study the stability of the zero solution to a suitable evolution problem in a Banach space. The pecularity of this problem is that 0 is an eigenvalue of the relevant linear operator, so that the classical "linearization principle" (e.g. [12], Sect. 5.1) does not apply. We need, instead, a "generalized linearization principle" in the spirit of Theorem 2.1 of [24]. Here, we shall follow the approach of [8], based on an operator fractional powers method, that appears to be more specific and direct for the type of fluid-structure interaction problems considered in this paper; see, however, also [19, 20]. In fact, the stability result stated in the following Theorem 3.2 is similar to its counterpart in Theorem 1.1 of [8], but obtained under slightly more general assumptions on the nonlinear operator and with a simpler argument. For completeness and reader's sake, in Theorem 3.3 we also provide a short proof of the complementary instability result.

In a (real) Banach space $X$, we consider the following evolution problem

$$
\frac{\mathrm{d} \boldsymbol{u}}{\mathrm{d} t}+\mathbf{L} \boldsymbol{u}=\mathbf{N}(\boldsymbol{u}), \quad \boldsymbol{u}(0) \in X
$$

with $\mathbf{L}$ and $\mathbf{N}$ to be defined next. Let $\mathbf{A}: X \mapsto X$ be a linear, sectorial operator with compact inverse and $\operatorname{Re} \sigma(\mathbf{A}) \subset(0, \infty)$. For $\alpha \in[0,1]$, set

$$
X_{\alpha}=\left\{\boldsymbol{u} \in X:\|\boldsymbol{u}\|_{\alpha}:=\left\|\mathbf{A}^{\alpha} \boldsymbol{u}\right\|<\infty\right\} ; \quad X_{0} \equiv X,\|\boldsymbol{u}\|_{0} \equiv\|\boldsymbol{u}\|
$$

It is well known that, for $\alpha>0, X_{\alpha}$ is a Banach space compactly embedded in $X$, e.g. Theorem 1.4.8 [12]. Let B : $X \mapsto X$ be a linear operator with $\mathrm{D}(\mathbf{B}) \supset \mathrm{D}(\mathbf{A})$, and such that

$$
\|\mathbf{B} \boldsymbol{u}\| \leq c_{1}\|\boldsymbol{u}\|_{\alpha}, \quad \alpha \in[0,1)
$$

We then assume

$$
\mathbf{L}=\mathbf{A}+\mathbf{B}
$$

with $D(\mathbf{L}) \equiv D(\mathbf{A})$. Since

$$
\|\boldsymbol{u}\|_{\alpha} \leq c\|\boldsymbol{u}\|^{1-\alpha}\|\mathbf{A} \boldsymbol{u}\|^{\alpha}
$$

and $\mathbf{A}^{-1}$ is compact, it follows that $\mathbf{B}$ is $\mathbf{A}$-compact, which in turn implies that $\mathbf{L}$ is an unbounded Fredholm operator of index 0 ([11], Thm. 4.3). Also, from ([12], Thm. 1.3.2) it follows, in particular, that $\mathbf{L}$ is sectorial. Finally, observing that by (3.4), for any $\varepsilon>0$,

$$
\|\mathbf{B} \boldsymbol{u}\| \leq c(\varepsilon)\|\boldsymbol{u}\|+\varepsilon\|\mathbf{A} \boldsymbol{u}\|
$$

and that, by the properties of $\mathbf{A}$,

$$
\left\|(\lambda+\mathbf{A})^{-1}\right\| \leq c_{2} \lambda^{-1}, \text { all } \lambda>0
$$

it follows ([13], Thm. 3.17 at p. 214) that $\mathbf{L}$ has a compact resolvent and, therefore, a discrete spectrum.

On the operator $\mathbf{L}$, we further assume:

$$
\operatorname{dim} \mathbf{N}[\mathbf{L}]=m \geq 1
$$




$$
\mathrm{N}[\mathbf{L}] \cap \mathrm{R}[\mathbf{L}]=\{\mathbf{0}\}
$$

and

$$
\sigma(\mathbf{L}) \cap\{\mathrm{i} \mathbb{R}\}=\{0\} .
$$

We then have the following.

Lemma 3.1. The space $X$ admits the decomposition

$$
X=\mathrm{N}[\mathbf{L}] \oplus \mathrm{R}[\mathbf{L}] .
$$

Moreover, denoting by $\mathcal{Q}$ and $\mathcal{P}$ the spectral projections according to the spectral sets

$$
\sigma_{0}(\mathbf{L}):=\{0\}, \quad \sigma_{1}(\mathbf{L}):=\sigma(\mathbf{L}) \backslash \sigma_{0}(\mathbf{L}),
$$

we have

$$
\mathrm{N}[\mathbf{L}]=\mathcal{Q}(X), \quad \mathrm{R}[\mathbf{L}]:=\mathcal{P}(X) .
$$

Finally, (3.5) completely reduces $\mathbf{L}$ into $\mathbf{L}=\mathbf{L}_{0} \oplus \mathbf{L}_{1}$ with

$$
\mathbf{L}_{0}:=\mathcal{Q} \mathbf{L}=\mathbf{L} \mathcal{Q}, \quad \mathbf{L}_{1}:=\mathcal{P} \mathbf{L}=\mathbf{L} \mathcal{P}
$$

and $\sigma\left(\mathbf{L}_{0}\right) \equiv \sigma_{0}(\mathbf{L}), \sigma\left(\mathbf{L}_{1}\right) \equiv \sigma_{1}(\mathbf{L})$.

Proof. Since $\mathbf{L}$ is Fredholm of index 0 , from (H1) we get $\operatorname{codim}(\mathrm{R}[\mathbf{L}])=m$. Thus, there exists at least one $S \subset X$ such that $X=S \oplus \mathrm{R}[\mathbf{L}]$, with $S \cap \mathrm{R}[\mathbf{L}]=\{\mathbf{0}\}$. However, $\operatorname{dim}(S)=\operatorname{dim}(\mathrm{N}[\mathbf{L}])=m$ and (H2) holds, so that we may take $S=\mathrm{N}[\mathbf{L}]$, which proves (3.5). The remaining properties stated in the lemma are then a consequence of (3.5) and classical results on spectral theory (e.g., [16], Prop. A.2.2; [30], Thms. 5.7-A,B)

We now turn to the operator $\mathbf{N}$. We begin to assume

$$
\begin{aligned}
& \left\|\mathbf{N}\left(\boldsymbol{u}_{1}\right)-\mathbf{N}\left(\boldsymbol{u}_{2}\right)\right\| \leq c\left\|\boldsymbol{u}_{1}-\boldsymbol{u}_{2}\right\|_{\alpha}, \text { for all } \boldsymbol{u}_{1}, \boldsymbol{u}_{2} \text { in a neighborhood of } \mathbf{0} \in X_{\alpha}, \\
& \|\mathbf{N}(\boldsymbol{u})\| \leq c\|\boldsymbol{u}\|_{\alpha}^{p}, \text { for some } p>1 \text { and all } \boldsymbol{u} \text { in a neighborhood of } \mathbf{0} \in X_{\alpha} .
\end{aligned}
$$

Furthermore, we observe that, by (3.5), every $\boldsymbol{u} \in X$ can be written as

$$
\boldsymbol{u}=\boldsymbol{u}^{(0)}+\boldsymbol{u}^{(1)}, \quad \boldsymbol{u}^{(0)} \in \mathrm{N}[\mathbf{L}], \boldsymbol{u}^{(1)} \in \mathrm{R}[\mathbf{L}] .
$$

Thus, setting

$$
\mathbf{M}\left(\boldsymbol{u}^{(0)}, \boldsymbol{u}^{(1)}\right):=\mathbf{N}\left(\boldsymbol{u}^{(0)}+\boldsymbol{u}^{(1)}\right)
$$

we suppose there is a non-negative, continuous function $\epsilon=\epsilon(\rho)$ with $\epsilon(0)=0$ such that

$$
\left\|\mathbf{M}\left(\boldsymbol{u}^{(0)}, \boldsymbol{u}^{(1)}\right)\right\| \leq \epsilon(\rho)\left\|\boldsymbol{u}^{(1)}\right\|_{\alpha},
$$


whenever $\left\|\boldsymbol{u}^{(0)}\right\|+\left\|\boldsymbol{u}^{(1)}\right\|_{\alpha} \leq C \rho$, some $C>0$.

We are now in a position to prove the following stability result; see also [8].

Theorem 3.2. Suppose the operators $\mathbf{L}$, defined in (3.3), and $\mathbf{N}$ satisfy hypotheses (H1)-(H4) 1 and (H5). Then, if $\operatorname{Re} \sigma\left(\mathbf{L}_{1}\right) \subset(0, \infty)$, we can find $\rho_{0}>0$ such that if

$$
\|\boldsymbol{u}(0)\|_{\alpha}<\rho_{0},
$$

there is a unique corresponding solution $\boldsymbol{u}=\boldsymbol{u}(t)$ to (3.1) for all $t>0$, satisfying, for any $T>0$,

$$
\boldsymbol{u} \in C\left([0, T) ; X_{\alpha}\right) \cap C\left((0, T) ; X_{1}\right) \cap C^{1}((0, T) ; X) .
$$

Moreover, the following two properties are satisfied:

(a) The solution $\boldsymbol{u}=\mathbf{0}$ to (3.1) is stable in $X_{\alpha}$, i.e., for any $\varepsilon>0$ there is $\delta>0$ such that

$$
\|\boldsymbol{u}(0)\|_{\alpha}<\delta \Longrightarrow \sup _{t \geq 0}\|\boldsymbol{u}(t)\|_{\alpha}<\varepsilon
$$

(b) There are $\eta, c, \kappa>0$ such that

$$
\|\boldsymbol{u}(0)\|_{\alpha}<\eta \Longrightarrow\|\boldsymbol{u}(t)-\overline{\boldsymbol{u}}\|_{\alpha} \leq c\left\|\boldsymbol{u}^{(1)}(0)\right\|_{\alpha} \mathrm{e}^{-\kappa t}, \text { all } t>0,
$$

for some $\overline{\boldsymbol{u}} \in \mathrm{N}[\mathbf{L}]$.

Proof. Under the stated assumptions on A, B and (H4) $)_{1}$, the existence of a unique solution $\boldsymbol{u}$ to (3.1) in some time interval $\left(0, t_{\star}\right)$ satisfying (3.8) for each $T \in\left(0, t_{\star}\right)$ is guaranteed by classical results on semilinear evolution equations (e.g., [23], p. 196-198). Moreover, $\left(0, t_{\star}\right)$ is maximal, in the sense that either $t_{\star}=\infty$ or else $\lim _{t \rightarrow t_{\star}}\|\boldsymbol{u}(t)\|_{\alpha}=\infty$. We shall next show that, in fact, only the former situation occurs for sufficiently "small" initial data. Applying $\mathcal{Q}$ and $\mathcal{P}$ on both sides of (3.1) and taking into account (3.7) we get

$$
\begin{aligned}
\frac{\mathrm{d} \boldsymbol{u}^{(1)}}{\mathrm{d} t}+\mathbf{L}_{1} \boldsymbol{u}^{(1)} & =\mathcal{P} \mathbf{M}\left(\boldsymbol{u}^{(0)}, \boldsymbol{u}^{(1)}\right) \\
\frac{\mathrm{d} \boldsymbol{u}^{(0)}}{\mathrm{d} t} & =\mathcal{Q M}\left(\boldsymbol{u}^{(0)}, \boldsymbol{u}^{(1)}\right),
\end{aligned}
$$

with $\boldsymbol{u}^{(0)}=\mathcal{Q} \boldsymbol{u}, \boldsymbol{u}^{(1)}=\mathcal{P} \boldsymbol{u}$. Since the operator $\mathbf{L}$, being sectorial, is the generator of an analytic semigroup in $X$, so is $\mathbf{L}_{1}$ in $X^{(1)} \equiv \mathrm{R}[\mathbf{L}]$. Thus, for all $t \in\left[0, t_{\star}\right)$ from $(3.9)_{1}$ we have

$$
\boldsymbol{u}^{(1)}(t)=\mathrm{e}^{-\mathbf{L}_{1} t} \boldsymbol{u}_{0}^{(1)}+\int_{0}^{t} \mathrm{e}^{-\mathbf{L}_{1}(t-s)}\left[\mathcal{P} \mathbf{M}\left(\boldsymbol{u}^{(0)}(s), \boldsymbol{u}^{(1)}(s)\right)\right] \mathrm{d} s .
$$

Also, by assumption and the spectral property of $\mathbf{L}$, there is $\gamma>0$ such that

$$
\operatorname{Re} \sigma\left(\mathbf{L}_{1}\right) \subset(\gamma, \infty),
$$

which implies that the fractional powers $\mathbf{L}_{1}^{\alpha}, \alpha \in(0,1)$, are well defined in $X^{(1)}$. Thus, setting

$$
\boldsymbol{w}:=\mathrm{e}^{b t} \mathbf{L}_{1}^{\alpha} \boldsymbol{u}^{(1)}, \quad 0<b<\gamma,
$$


from (3.10) we get

$$
\boldsymbol{w}(t)=\mathrm{e}^{b t} \mathrm{e}^{-\mathbf{L}_{1} t} \mathbf{L}_{1}^{\alpha} \boldsymbol{u}_{0}^{(1)}+\int_{0}^{t} \mathrm{e}^{b t} \mathbf{L}_{1}^{\alpha} \mathrm{e}^{-\mathbf{L}_{1}(t-s)}\left[\mathcal{P} \mathbf{M}\left(\boldsymbol{u}^{(0)}(s), \mathrm{e}^{-b s} \mathbf{L}_{1}^{-\alpha} \boldsymbol{w}(s)\right] \mathrm{d} s .\right.
$$

From the local existence theory considered earlier on, we know that for any given $\rho>0$ there exists an interval of time $[0, \tau], \tau<t_{\star}$, such that

$$
\sup _{t \in[0, \tau)}\left(\|\boldsymbol{w}(t)\|+\left\|\boldsymbol{u}^{(0)}(t)\right\|\right) \leq \rho, \tau<t_{\star}
$$

provided $\|\boldsymbol{u}(0)\|_{\alpha}<\eta$, for some $\eta>0$. Let us show that $\eta$ and $\rho$ can be chosen sufficiently small so that (3.13) holds also with $\tau=t_{\star}$, thus implying, in particular, that the solution $\boldsymbol{u}=\boldsymbol{u}(t)$ to (3.1) exists for all times $t>0$. In fact, suppose, by contradiction, that there is $\tau_{0}<t_{\star}$ such that

$$
\|\boldsymbol{w}(t)\|+\left\|\boldsymbol{u}^{(0)}(t)\right\|<\rho, \quad t \in\left[0, \tau_{0}\right) \quad \text { and } \quad\left\|\boldsymbol{w}\left(\tau_{0}\right)\right\|+\left\|\boldsymbol{u}^{(0)}\left(\tau_{0}\right)\right\|=\rho .
$$

In view of the stated properties of $\mathbf{L}_{1}$ it results (e.g. [12], Thm. 1.4.6),

$$
\left\|\mathbf{L}_{1}^{-\alpha} \boldsymbol{w}\right\|_{\alpha} \leq c_{1}\|\boldsymbol{w}\|, \text { for all } \boldsymbol{w} \in X^{(1)},
$$

Moreover, we recall that in $X^{(1)}$ it is

$$
\left\|\mathbf{L}_{1}^{\alpha} \mathrm{e}^{-\mathbf{L}_{1} t}\right\| \leq C_{\alpha} t^{-\alpha} \mathrm{e}^{-\gamma t}
$$

and observe that from (3.2) and ([12], Thm. 1.4.6)

$$
\left\|\mathbf{L}_{1}^{\alpha} \boldsymbol{u}_{0}^{(1)}\right\| \leq c_{2}\left\|\boldsymbol{u}_{0}^{(1)}\right\|_{\alpha}
$$

Thus, noticing that

$$
\int_{0}^{t} \frac{\mathrm{e}^{-(\gamma-b)(t-s)}}{(t-s)^{\alpha}} \mathrm{d} s \leq \int_{0}^{\infty} \frac{\mathrm{e}^{-(\gamma-b) t}}{t^{\alpha}} d t<\infty,
$$

from (3.12), (3.14)-(3.17), and (H5) we show

$$
\|\boldsymbol{w}(t)\| \equiv \mathrm{e}^{b t}\left\|\boldsymbol{u}^{(1)}(t)\right\|_{\alpha} \leq C_{0} \eta+\epsilon(\rho) \rho, \text { for all } t \in\left[0, \tau_{0}\right]
$$

On the other hand, $(3.9)_{2}$, with the help of (3.14), (H5) and (3.18), furnishes

$$
\begin{aligned}
\left\|\boldsymbol{u}^{(0)}(t)\right\| \leq & \left\|\boldsymbol{u}^{(0)}(0)\right\|+\epsilon(\rho) \int_{0}^{t}\left\|\boldsymbol{u}^{(1)}(s)\right\|_{\alpha} \mathrm{d} s \leq\left\|\boldsymbol{u}^{(0)}(0)\right\|+\epsilon(\rho) \int_{0}^{t} \mathrm{e}^{-b s}\|\boldsymbol{w}(s)\| \mathrm{d} s \\
& \leq c_{3} \eta+\epsilon(\rho) \rho, \quad t \in\left[0, \tau_{0}\right] .
\end{aligned}
$$

Combining (3.18) and (3.19), and choosing $c_{3} \eta / \rho+\epsilon(\rho)<1 / 4$ we conclude in particular

$$
\left\|\boldsymbol{w}\left(\tau_{0}\right)\right\|+\left\|\boldsymbol{u}^{(0)}\left(\tau_{0}\right)\right\| \leq \rho / 2
$$


contradicting (3.14). As a result, by what we observed early on, we may take $t_{\star}=\infty$ in (3.13) and conclude as well

$$
\sup _{t \in[0, \infty)}\left(\|\boldsymbol{w}(t)\|+\left\|\boldsymbol{u}^{(0)}(t)\right\|\right) \leq \rho
$$

proving, as a byproduct, the desired global existence property. Now, employing in (3.12), the inequalities (3.20), (3.15) along with the assumption (H5), we easily deduce, for $\rho$ small enough,

$$
\|\boldsymbol{w}(t)\| \leq c_{4}\left\|\boldsymbol{u}^{(1)}(0)\right\|_{\alpha}, \text { all } t>0
$$

namely,

$$
\left\|\boldsymbol{u}^{(1)}(t)\right\|_{\alpha} \leq c_{4} \mathrm{e}^{-b t}\left\|\boldsymbol{u}^{(1)}(0)\right\|_{\alpha}, \text { all } t>0 .
$$

Also, using (3.21) into (3.19), we infer

$$
\left\|\boldsymbol{u}^{(0)}(t)\right\| \leq c_{5}\|\boldsymbol{u}(0)\|_{\alpha}, \text { all } t>0
$$

Therefore, from (3.22) and (3.23) we recover the stability property stated in (a). Moreover, integrating (3.9) ${ }_{2}$ between arbitrary $t_{1}, t_{2}>0$ using (3.22) and reasoning in a way similar to what we did to obtain (3.19) we get

$$
\left\|\boldsymbol{u}^{(0)}\left(t_{1}\right)-\boldsymbol{u}^{(0)}\left(t_{2}\right)\right\| \leq c_{6} \int_{t_{1}}^{t_{2}}\left\|\boldsymbol{u}^{(1)}(s)\right\|_{\alpha} \mathrm{d} s \leq c_{7}\left\|\boldsymbol{u}^{(1)}(0)\right\|_{\alpha} \int_{t_{1}}^{t_{2}} \mathrm{e}^{-b s} \mathrm{~d} s
$$

from which we deduce that there exists $\overline{\boldsymbol{u}} \in \mathrm{N}[\mathbf{L}]$ such that

$$
\lim _{t \rightarrow \infty}\left\|\boldsymbol{u}^{(0)}(t)-\overline{\boldsymbol{u}}\right\|=0 .
$$

Employing this information into (3.24) in the limit $t_{2} \rightarrow \infty$, and with $t_{1}=t$ we show

$$
\left\|\boldsymbol{u}^{(0)}(t)-\overline{\boldsymbol{u}}\right\|_{\alpha} \leq c_{8}\left\|\boldsymbol{u}^{(0)}(t)-\overline{\boldsymbol{u}}\right\| \leq c_{9}\left\|\boldsymbol{u}^{(1)}(0)\right\|_{\alpha} \mathrm{e}^{-b t}
$$

which, once combined with (3.22), proves the exponential rate of decay stated in (b). The proof of the theorem is thus concluded.

We next show the following instability result. ${ }^{2}$

Theorem 3.3. Let $\mathbf{L}$ be the operator defined in (3.3), and suppose that $\mathbf{N}$ satisfies hypotheses (H1)-(H4) Then, if $\operatorname{Re} \sigma(\mathbf{L}) \cap(-\infty, 0) \neq \emptyset$ the solution $\boldsymbol{u}=\mathbf{0}$ to (3.1) is unstable in $X_{\alpha}$. Precisely, there is $\varepsilon_{0}>0$ with the following property: for any given $\delta \in(0,1]$ there is a solution $\boldsymbol{u}(t)$ to (3.1) in the class (3.8) with $\|\boldsymbol{u}(0)\|_{\alpha} \leq \delta$ such that

$$
\sup _{t \in\left(0, t^{*}\right)}\|\boldsymbol{u}(t)\|_{\alpha} \geq \varepsilon_{0}
$$

where $\left(0, t^{*}\right)$ is the maximal interval of existence.

\footnotetext{
${ }^{2}$ The results stated in Theorem 3.3 could also be obtained by means of Theorem 5.1 .5 in [12]. However, for completeness, we present a simple proof by a different method.
} 
Proof. We follow the ideas of Theorem 2.3 in Chapter VII in [3]. From (3.1) we deduce

$$
\boldsymbol{u}(t)=\mathrm{e}^{-\mathbf{L} t} \boldsymbol{u}(0)+\int_{0}^{t} \mathrm{e}^{-\mathbf{L}(t-s)} \mathbf{N}(\boldsymbol{u}(s)) \mathrm{d} s:=\boldsymbol{u}_{1}(t)+\boldsymbol{u}_{2}(t) .
$$

Let $\lambda_{0}$ be the eigenvalue of $\mathbf{L}$ such that

$$
-a_{0}:=\operatorname{Re} \lambda_{0}=\min _{\lambda \in \sigma(\mathbf{L})} \operatorname{Re} \lambda .
$$

By assumption, $a_{0}>0$ and we write $\lambda_{0}=-a_{0}+\mathrm{i} b_{0}, b_{0} \in \mathbb{R}$. Let $\varphi \in \mathrm{D}(\mathbf{L})(\equiv \mathrm{D}(\mathbf{A}))$ be the eigenvector corresponding to $\lambda_{0}$, normalized with $\|\boldsymbol{\varphi}\|_{\alpha}=1$, and choose $\boldsymbol{u}(0)=\delta \operatorname{Re} \boldsymbol{\varphi}$. Since $\mathrm{e}^{-\mathbf{L} t} \boldsymbol{u}(0)=\delta \mathrm{e}^{\left(a_{0}+\mathrm{i} b_{0}\right) t} \boldsymbol{\varphi}$ we get, therefore,

$$
\left\|\boldsymbol{u}_{1}(t)\right\|_{\alpha} \leq \delta \mathrm{e}^{a_{0} t}
$$

Next, fix $R \in(1,2)$ and denote by $\tau_{0}\left(<t^{*}\right)$ the largest positive number for which

$$
\|\boldsymbol{u}(t)\|_{\alpha} \leq \delta R \mathrm{e}^{a_{0} t}, \quad t \in\left[0, \tau_{0}\right]
$$

Let $\mathbf{L}_{\zeta}:=\mathbf{L}+\zeta$, with $\zeta>a_{0}$. Clearly, $\operatorname{Re} \sigma\left(\mathbf{L}_{\zeta}\right) \subset(0, \infty)$, so that $\mathbf{L}_{\zeta}^{-\alpha}$ is well defined. We thus get

$$
\mathbf{A}^{\alpha} \mathrm{e}^{-\mathbf{L}(t-s)} \mathbf{N}(\boldsymbol{u}(s))=\mathrm{e}^{\zeta(t-s)} \mathbf{A}^{\alpha} \mathbf{L}_{\zeta}^{-\alpha} \mathbf{L}_{\zeta}^{\alpha} \mathrm{e}^{-\mathbf{L}_{\zeta}(t-s)} \mathbf{N}(\boldsymbol{u}(s))
$$

which, in turn, after observing that $\mathbf{A}^{\alpha} \mathbf{L}_{\zeta}^{-\alpha}$ is bounded in $X$ ([12], Thm. 1.4.6), and taking into account (H4) $)_{2}$, implies

$$
\left\|\mathbf{A}^{\alpha} \mathrm{e}^{-\mathbf{L}(t-s)} \mathbf{N}(\boldsymbol{u}(s))\right\| \leq C \mathrm{e}^{\zeta(t-s)}\left\|\mathbf{L}_{\zeta}^{\alpha} \mathrm{e}^{-\mathbf{L}_{\zeta}(t-s)}\right\|\|\boldsymbol{u}(s)\|_{\alpha}^{p} .
$$

Therefore, since

$$
\left\|\mathbf{L}_{\zeta}^{\alpha} \mathrm{e}^{-\mathbf{L}_{\zeta}(t-s)}\right\| \leq c_{\alpha} \frac{\mathrm{e}^{\left(a_{0}-\zeta\right)(t-s)}}{(t-s)^{\alpha}}
$$

from (3.25), and (3.27)-(3.29) we show

$$
\begin{aligned}
\left\|\boldsymbol{u}_{2}(t)\right\|_{\alpha} & \leq c_{1} \int_{0}^{t} \mathrm{e}^{\zeta(t-s)} \frac{\mathrm{e}^{\left(a_{0}-\zeta\right)(t-s)}}{(t-s)^{\alpha}}\|\boldsymbol{u}(s)\|_{\alpha}^{p} \mathrm{~d} s=c_{1} \int_{0}^{t} \frac{\mathrm{e}^{a_{0}(t-s)}}{(t-s)^{\alpha}}\|\boldsymbol{u}(s)\|_{\alpha}^{p} \mathrm{~d} s \\
& \leq c_{1} \delta^{p} R^{p} \mathrm{e}^{p a_{0} t} \int_{0}^{t} \frac{\mathrm{e}^{a_{0}(1-p) \rho}}{\rho^{\alpha}} d \rho \leq c_{0} \delta^{p} R^{p} \mathrm{e}^{p a_{0} t}, \quad t \in\left[0, \tau_{0}\right],
\end{aligned}
$$

where $c_{0}=c_{0}\left(\alpha, a_{0}\right)$. Define the positive number $\tau$ through the relation

$$
1+c_{0} \delta^{p-1} R^{p} \mathrm{e}^{(p-1) a_{0} \tau}=R .
$$


It is clear that $\tau \leq \tau_{0}$, because otherwise from (3.25), (3.26), (3.30) and (3.31) we would contradict the definition of $\tau_{0}$ for which (3.27) holds. However, for this $\tau$, from (3.25), (3.26), (3.30) and (3.31) it follows that

$$
\|\boldsymbol{u}(\tau)\|_{\alpha} \geq \delta \mathrm{e}^{a_{0} \tau}\left(1-c_{0} \delta^{p-1} R^{p} \mathrm{e}^{(p-1) a_{0} \tau}\right)=\left(\frac{R-1}{c_{0} R^{p}}\right)^{\frac{1}{p-1}}(2-R):=\varepsilon_{0} .
$$

The proof of the theorem is completed.

\section{Preliminary properties of the operator L}

The main purpose of this section is to find conditions under which the operator $\mathbf{L}$ defined in $(2.11),(2.10)$ satisfies the assumptions (H1)-(H3) stated in the previous section. In this regard we begin to observe that, by the well known properties of the Stokes operator

$$
\boldsymbol{A}_{0}:=-\nu \mathrm{P} \Delta
$$

with domain $\mathrm{D}\left(\boldsymbol{A}_{0}\right):=W^{2,2}(\mathcal{C}) \cap \mathcal{D}_{0}^{1,2}(\mathcal{C})$ and range $L_{\sigma}^{2}(\mathcal{C})$, it follows that $\boldsymbol{A}$ has a compact inverse and, therefore, a discrete spectrum which, in addition, lies on the positive real axis. Since $\boldsymbol{I}^{-1}$ is symmetric (and bounded), the operator $\mathbf{A}$ enjoys the same properties as $\boldsymbol{A}$. Furthermore, $\boldsymbol{B}$ is bounded and therefore $\mathbf{B}$ satisfies (3.2) with $\alpha=0$. We shall now check the validity of the other assumptions.

We start with the following result which, in particular, gives sufficient conditions in order that $\mathbf{L}$ satisfies (H1).

Proposition 4.1. Let $\overline{\mathrm{s}}$ be the steady-state solution (2.4) and let $\mathbf{L}=\mathbf{L}(\overline{\boldsymbol{s}})$ be the linearization around $\overline{\mathrm{s}}$. Then, setting

$$
K_{A}:=\frac{\lambda^{2}}{\beta^{2}}(C-A), \quad K_{B}:=\frac{\lambda^{2}}{\beta^{2}}(C-B)
$$

we have

$$
\mathrm{N}[\mathbf{L}]=\left\{\boldsymbol{v} \equiv \mathbf{0} ; \boldsymbol{\omega}=r \boldsymbol{e}_{3} ; \boldsymbol{z}=z \boldsymbol{e}_{3} ; \quad(r, z) \in \mathbb{R}^{2}\right\}, \quad \operatorname{dim} \mathrm{N}[\mathbf{L}]=2,
$$

provided $K_{A}, K_{B} \neq 1$.

Proof. From Lemma 3.1 and $(2.10)_{2,3}$, it follows that the equation $\mathbf{L} \boldsymbol{u}=\mathbf{0}$ is equivalent to the following system of equations

$$
\begin{aligned}
& \left.\begin{array}{l}
\nu \Delta \boldsymbol{v}-\nabla p=-2 \lambda \boldsymbol{e}_{3} \times \boldsymbol{v} \\
\operatorname{div} \boldsymbol{v}=0
\end{array}\right\} \text { in } \mathcal{C}, \\
& \boldsymbol{v}=\mathbf{0} \quad \text { on } \partial \mathcal{C}, \\
& -\lambda e_{3} \times \mathbb{I} \cdot(\boldsymbol{\omega}-\boldsymbol{a})+\lambda C \boldsymbol{e}_{3} \times \boldsymbol{\omega}=\beta^{2} \boldsymbol{e}_{3} \times \boldsymbol{z}, \\
& -\boldsymbol{\omega} \times \boldsymbol{e}_{3}-\lambda e_{3} \times \boldsymbol{z}=\mathbf{0}
\end{aligned}
$$

By dot-multiplying both sides of $(4.4)_{1}$ by $\boldsymbol{v}$, integrating by parts over $\mathcal{C}$ and using $(4.4)_{2,3}$, we deduce $\boldsymbol{v} \equiv \mathbf{0}$. As a result, the elements of $\mathrm{N}[\mathbf{L}]$ must have $\boldsymbol{v} \equiv \mathbf{0}$, while $\boldsymbol{\omega}$ and $\boldsymbol{z}$ solve the following equations

$$
\begin{aligned}
& -\lambda \boldsymbol{e}_{3} \times \mathbb{I} \cdot \boldsymbol{\omega}+\boldsymbol{e}_{3} \times\left(\lambda C \boldsymbol{\omega}-\beta^{2} \boldsymbol{z}\right)=\mathbf{0}, \\
& \boldsymbol{\omega}=\lambda \boldsymbol{z}+\mu \boldsymbol{e}_{3}, \quad \mu \in \mathbb{R} .
\end{aligned}
$$


Replacing $(4.5)_{2}$ into $(4.5)_{1}$ and recalling that $\mathbb{I}=\operatorname{diag}(A, B, C)$ we thus get

$$
\left(1-K_{B}\right) z_{2} \boldsymbol{e}_{1}+\left(K_{A}-1\right) z_{1} \boldsymbol{e}_{2}=\mathbf{0},
$$

which, in turn, under the given assumptions furnishes $\boldsymbol{z}=z \boldsymbol{e}_{3}, z \in \mathbb{R}$. The desired property is then a consequence of the latter and of $(4.5)_{2}$.

We now pass to the investigation of the validity of the assumption (H2).

Proposition 4.2. Let the assumptions of Proposition 4.1 be satisfied. Then $N[\mathbf{L}] \cap R[\mathbf{L}]=\{\mathbf{0}\}$.

Proof. From (2.10), (2.11) and Proposition 4.1, we deduce that the condition $N[\mathbf{L}] \cap R[\mathbf{L}]=\{\mathbf{0}\}$ is equivalent to show that, if $\left(\boldsymbol{v}_{0} \equiv 0, \boldsymbol{\omega}_{0}=\omega_{0} \boldsymbol{e}_{3}, \boldsymbol{z}_{0}=z_{0} \boldsymbol{e}_{3}\right) \in \mathrm{N}[\mathbf{L}]$, and

$$
\begin{aligned}
& \left.\begin{array}{l}
-\nu \Delta \boldsymbol{v}-2 \lambda \boldsymbol{e}_{3} \times \boldsymbol{v}+\nabla p=\omega_{0} \boldsymbol{e}_{3} \times \boldsymbol{x} \\
\operatorname{div} \boldsymbol{v}=0
\end{array}\right\} \text { in } \mathcal{C}, \\
& \boldsymbol{v}=\mathbf{0} \text { at } \partial \mathcal{C} \\
& -\lambda \boldsymbol{e}_{3} \times \mathbb{I} \cdot(\boldsymbol{\omega}-\boldsymbol{a})-\lambda C \boldsymbol{\omega} \times \boldsymbol{e}_{3}-\beta^{2} \boldsymbol{e}_{3} \times \boldsymbol{z}=C \omega_{0} \boldsymbol{e}_{3} \\
& (\boldsymbol{\omega}-\lambda \boldsymbol{z}) \times \boldsymbol{e}_{3}=-z_{0} \boldsymbol{e}_{3}
\end{aligned}
$$

for some $(\boldsymbol{v}, \boldsymbol{\omega}, \boldsymbol{z}) \in \mathrm{D}(\boldsymbol{A})$, then necessarily $\boldsymbol{\omega}_{0}=\boldsymbol{z}_{0}=\mathbf{0}$. However, the latter follows at once by dot-multiplying both sides of $(4.6)_{4,5}$ by $\boldsymbol{e}_{3}$.

In order to investigate further properties of the operator $\mathbf{L}$, we need the following.

Lemma 4.3. Let

$$
\mathcal{G}=\mathcal{G}(\boldsymbol{u}):=\|\boldsymbol{v}\|_{2}^{2}-\boldsymbol{a} \cdot \mathbb{I} \cdot \boldsymbol{a}+\boldsymbol{\omega}_{*} \cdot \mathbb{I} \cdot \boldsymbol{\omega}_{*}+\delta|\boldsymbol{z}|^{2}-2 \lambda \boldsymbol{z} \cdot \mathbb{I} \cdot \boldsymbol{\omega}_{*}
$$

where $\boldsymbol{\omega}_{*}:=\boldsymbol{\omega}-\boldsymbol{a}$ (with $\boldsymbol{a}$ given in (2.2)) and

$$
\delta=\lambda^{2} C-\beta^{2} .
$$

Then

$$
\frac{1}{2} \frac{\mathrm{d} \mathcal{G}}{\mathrm{d} t}+\nu\|\nabla \boldsymbol{v}\|_{2}^{2}=0
$$

along the solutions to the linear problem

$$
\frac{\mathrm{d} \boldsymbol{u}}{\mathrm{d} t}+\mathbf{L} \boldsymbol{u}=\mathbf{0}, \quad \boldsymbol{u}(0) \in X
$$


Proof. From (2.12) we deduce that the evolution equation in (4.10) is equivalent to the following set of equations:

$$
\left.\begin{array}{l}
\left.\begin{array}{l}
\boldsymbol{v}_{t}+\left(\dot{\boldsymbol{\omega}}_{*}+\dot{\boldsymbol{a}}\right) \times \boldsymbol{x}-2 \lambda \boldsymbol{e}_{3} \times \boldsymbol{v}=\nu \Delta \boldsymbol{v}-\nabla p \\
\operatorname{div} \boldsymbol{v}=0
\end{array}\right\} \text { on } \mathcal{C} \times(0, \infty), \\
\mathbb{I} \cdot \dot{\boldsymbol{\omega}}_{*}-\lambda \boldsymbol{e}_{3} \times \mathbb{I} \cdot \boldsymbol{\omega}_{*}-\lambda C\left(\boldsymbol{\omega}_{*}+\boldsymbol{a}\right) \times \boldsymbol{e}_{3}=\beta^{2} \boldsymbol{e}_{3} \times \boldsymbol{z} \\
\dot{\boldsymbol{z}}-\left(\boldsymbol{\omega}_{*}+\boldsymbol{a}\right) \times \boldsymbol{e}_{3}-\lambda \boldsymbol{e}_{3} \times \boldsymbol{z}=\mathbf{0} \\
\boldsymbol{v}=\mathbf{0} \text { on } \partial \mathcal{C} \times(0, \infty)
\end{array}\right\} \text { in }(0, \infty),
$$

Due to the analyticity of the semigroup generated by $\mathbf{L}$, solutions to (4.11) with initial data in $X$ are smooth. We dot-multiply both sides of $(4.11)_{1}$ by $\boldsymbol{v}$ and integrate by parts over $\mathcal{C}$ to get

$$
\frac{1}{2}\left(\frac{\mathrm{d}}{\mathrm{d} t}\|\boldsymbol{v}\|_{2}^{2}-\boldsymbol{a} \cdot \mathbb{I} \cdot \boldsymbol{a}\right)-\dot{\boldsymbol{\omega}}_{*} \cdot \mathbb{I} \cdot \boldsymbol{a}+\nu\|\nabla \boldsymbol{v}\|_{2}^{2}=0 .
$$

We next dot-multiply both sides of $(4.11)_{3}$ by $\boldsymbol{z}$ and both sides of $(4.11)_{4}$ by $\mathbb{I} \cdot \boldsymbol{\omega}_{*}$, and sum the resulting equations side-by-side. We thus infer

$$
\frac{\mathrm{d}}{\mathrm{d} t}\left(\boldsymbol{z} \cdot \mathbb{I} \cdot \boldsymbol{\omega}_{*}\right)-\left(\boldsymbol{\omega}_{*}+\boldsymbol{a}\right) \times \boldsymbol{e}_{3} \cdot\left(\mathbb{I} \cdot \boldsymbol{\omega}_{*}\right)-\lambda C\left(\boldsymbol{\omega}_{*}+\boldsymbol{a}\right) \times \boldsymbol{e}_{3} \cdot \boldsymbol{z}=0 .
$$

By dot-multiplying both sides of $(4.11)_{3}$ by $\boldsymbol{\omega}_{*}+\boldsymbol{a}$ we show

$$
\frac{1}{2} \frac{\mathrm{d}}{\mathrm{d} t}\left(\boldsymbol{\omega}_{*} \cdot \mathbb{I} \cdot \boldsymbol{\omega}_{*}\right)-\lambda \boldsymbol{e}_{3} \times\left(\mathbb{I} \cdot \boldsymbol{\omega}_{*}\right) \cdot\left(\boldsymbol{\omega}_{*}+\boldsymbol{a}\right)+\boldsymbol{a} \cdot \mathbb{I} \cdot \dot{\boldsymbol{\omega}}_{*}-\beta^{2} \boldsymbol{e}_{3} \times \boldsymbol{z} \cdot\left(\boldsymbol{\omega}_{*}+\boldsymbol{a}\right)=0 .
$$

Finally, dot-multiplying both sides of $(4.11)_{4}$ by $\boldsymbol{z}$ we obtain

$$
\frac{1}{2} \frac{\mathrm{d}}{\mathrm{d} t}|\boldsymbol{z}|^{2}-\left(\boldsymbol{\omega}_{*}+\boldsymbol{a}\right) \cdot \boldsymbol{e}_{3} \times \boldsymbol{z}=0 .
$$

If we form the linear combination $(4.12)+(4.14)+\delta(4.15)-\lambda(4.13)$ and use (4.8), we deduce

$$
\frac{1}{2} \frac{\mathrm{d} \mathcal{G}}{\mathrm{d} t}+\nu\|\nabla \boldsymbol{v}\|_{2}^{2}=\left(\boldsymbol{\omega}_{*}+\boldsymbol{a}\right) \cdot\left[\left(\beta^{2} \boldsymbol{e}_{3}+\delta \boldsymbol{e}_{3}-\lambda^{2} C \boldsymbol{e}_{3}\right) \times \boldsymbol{z}\right]=0
$$

which completes the proof.

A first important consequence of this lemma is provided by the following result that furnishes sufficient conditions for the validity of (H3).

Proposition 4.4. $\sigma(\mathbf{L}) \cap\{\mathrm{i} \mathbb{R}\}=\{0\}$.

Proof. Contradicting the stated property means that the system (4.11) has at least one time-periodic solution $\left(\boldsymbol{v}, \boldsymbol{\omega}_{*}, \boldsymbol{z}\right)$ of period $T>0$ (say) such that

$$
\int_{0}^{T} \boldsymbol{v}(x, t) \mathrm{d} t=\int_{0}^{T} \boldsymbol{\omega}_{*}(t) \mathrm{d} t=\int_{0}^{T} \boldsymbol{z}(t) \mathrm{d} t=\mathbf{0} .
$$


Integrating (4.9) from 0 to $T$ and using the periodicity we deduce

$$
\int_{0}^{T}\|\nabla \boldsymbol{v}\|_{2}^{2} \mathrm{~d} t=0
$$

which, in turn, by $(4.11)_{5}$ furnishes $\boldsymbol{v} \equiv \mathbf{0}$. Then, by $(4.11)_{1}$ it is $\dot{\boldsymbol{\omega}}_{*}=-\nabla p$ and by operating with curl on both sides we get $\boldsymbol{\omega}_{*}=$ const, implying, by (4.16), $\boldsymbol{\omega}_{*}=\mathbf{0}$. The latter in conjunction with (4.11) 3 delivers $\boldsymbol{z}=z_{3} \boldsymbol{e}_{3}$ that, once combined with $(4.11)_{4}$, allows us to deduce $z_{3}=$ const. Thus, by (4.16) we find also that $\boldsymbol{z}=\mathbf{0}$, and the proof of the proposition is completed.

We conclude this section by collecting in the following theorem some relevant consequences of Proposition 4.1, Proposition 4.2 and Proposition 4.4.

Theorem 4.5. Suppose the assumptions of Proposition 4.1 are satisfied. Then, the linear operator $\mathbf{L}=\mathbf{L}(\overline{\mathbf{s}})$ meets all conditions (H1)-(H3).

In the next section we shall give detailed information on the spectrum of $\mathbf{L}$.

\section{ON THE LOCATION OF THE SPECTRUM OF THE OPERATOR L}

We now turn to the study of the location of the eigenvalues of the operator $\mathbf{L}$. As a matter of fact, according to the decomposition proved in Lemma 3.1, this amounts to investigate the same property for the restriction, $\mathbf{L}_{1}$, of $\mathbf{L}$ to the subspace $R[\mathbf{L}]$.

To this end, we propose two preparatory results collected in the form of as many lemmas.

Lemma 5.1. Suppose the assumptions of Proposition 4.1 hold, and that the number $\delta$, defined in (4.8), is such that

$$
\delta>\lambda^{2} \max \{A, B\} .
$$

Then, all solutions $\boldsymbol{u}$ to problem (4.10) satisfy the estimate

$$
\sup _{t \geq 0}\|\boldsymbol{u}(t)\| \leq \kappa
$$

for some $\kappa=\kappa(\boldsymbol{u}(0), A, B, C)>0$.

Proof. Setting $\boldsymbol{\omega}_{*}=\left(\zeta_{1}, \zeta_{2}, \zeta_{3}\right)$, we define

$$
\begin{aligned}
& Q_{1}=Q_{1}\left(\zeta_{1}, z_{1}\right):=A \zeta_{1}^{2}+\delta z_{1}^{2}-2 \lambda A \zeta_{1} z_{1} \\
& \mathcal{Q}_{2}=\mathcal{Q}_{2}\left(\zeta_{2}, z_{2}\right):=B \zeta_{2}^{2}+\delta z_{2}^{2}-2 \lambda B \zeta_{2} z_{2} \\
& \mathcal{Q}_{3}=Q_{3}\left(\zeta_{3}, z_{3}\right):=C \zeta_{3}^{2}+\delta z_{3}^{2}-2 \lambda C \zeta_{3} z_{3},
\end{aligned}
$$

so that the functional $\mathcal{G}$ in $(4.7)$ can be written as

$$
\mathcal{G}=\|\boldsymbol{v}\|_{2}^{2}-\boldsymbol{a} \cdot \mathbb{I} \cdot \boldsymbol{a}+Q_{1}+Q_{2}+Q_{3} .
$$

We next observe that by Sections 7.2.2, 7.2.3 of [14], there is $\kappa_{0} \in(0,1)$ such that

$$
\kappa_{0}\|\boldsymbol{v}\|_{2}^{2} \leq \mathcal{E}_{F}:=\|\boldsymbol{v}\|_{2}^{2}-\boldsymbol{a} \cdot \mathbb{I} \cdot \boldsymbol{a} \leq\|\boldsymbol{v}\|_{2}^{2} .
$$


Now, since $\delta>\lambda^{2} \max \{A, B, C\}$, all quadratic form $Q_{i}, i=1,2,3$, are positive definite. As a consequence, integrating both sides of (4.9) from 0 to $t$ and using this information along with (5.5) leads to (5.2).

Lemma 5.2. Let $\boldsymbol{u}$ be a solution to (4.10) with initial data $\boldsymbol{u}(0)=\boldsymbol{u}_{0}$, such that

$$
\sup _{t \geq 0}\|\boldsymbol{u}(t)\| \leq \kappa,
$$

for some constant $\kappa>0$. Then, the corresponding $\omega$-limit set, $\Omega\left(\boldsymbol{u}_{0}\right)$ is not empty and satisfies

$$
\Omega\left(\boldsymbol{u}_{0}\right) \subseteq \mathrm{N}[\mathbf{L}] .
$$

Proof. We recall that (4.10) is equivalent to (4.11) endowed with the appropriate initial data. Thus, from (5.6) and $(4.11)_{3}$, it also follows that $\left|\dot{\boldsymbol{\omega}}_{*}\right|$ is uniformly bounded in time. Therefore (4.12), together with Poincaré inequality and (5.5), implies

$$
\frac{\mathrm{d}}{\mathrm{d} t} \mathcal{E}_{F}(\boldsymbol{v})+c_{1} \mathcal{E}_{F}(\boldsymbol{v}) \leq c_{2}\|\boldsymbol{v}\|_{2}
$$

Furthermore, by (4.9), (5.6) and Poincaré inequality, it follows that $\boldsymbol{v} \in L^{2}\left(0, \infty ; L_{\sigma}^{2}(\mathcal{C})\right)$. The latter, combined with (5.7) and (5.5), allows us to conclude that (see [18], Lem. 2.3.4)

$$
\lim _{t \rightarrow \infty}\|\boldsymbol{v}(t)\|_{2}=0
$$

From this and again (5.6), it follows that the $\omega$-limit set $\Omega\left(\boldsymbol{u}_{0}\right)$ of the dynamical system generated by (4.10) must be connected, compact and invariant. Moreover,

$$
\lim _{t \rightarrow \infty} \operatorname{dist}\left(\boldsymbol{u}, \Omega\left(\boldsymbol{u}_{0}\right)\right)=0
$$

The dynamics on the $\omega$-limit set is then characterized by $\boldsymbol{v} \equiv \mathbf{0}$, and $\boldsymbol{\omega} \equiv \boldsymbol{\omega}_{*}$, and $\boldsymbol{z}$ satisfying

$$
\begin{aligned}
& \dot{\boldsymbol{\omega}} \times \boldsymbol{x}=-\nabla p \\
& \mathbb{I} \cdot \dot{\boldsymbol{\omega}}-\lambda \boldsymbol{e}_{3} \times \mathbb{I} \cdot \boldsymbol{\omega}-\lambda C \boldsymbol{\omega} \times \boldsymbol{e}_{3}=\beta^{2} \boldsymbol{e}_{3} \times \boldsymbol{z}, \\
& \dot{\boldsymbol{z}}-\boldsymbol{\omega} \times \boldsymbol{e}_{3}-\lambda C \boldsymbol{e}_{3} \times \boldsymbol{z}=\mathbf{0} .
\end{aligned}
$$

By taking the curl of both sides of the first equation, it immediately follows $\dot{\boldsymbol{\omega}}=\mathbf{0}$, which, in turn, by the second equation, furnishes $\dot{z}_{i}=0, i=1,2$. Using this information in the third equation dot-multiplied by $\boldsymbol{e}_{3}$ we get also $\dot{z}_{3}=0$. As a consequence, the previous system reduces to

$$
\begin{aligned}
& -\lambda \boldsymbol{e}_{3} \times \mathbb{I} \cdot \boldsymbol{\omega}+\boldsymbol{e}_{3} \times\left(\lambda C \boldsymbol{\omega}-\beta^{2} \boldsymbol{z}\right)=\mathbf{0}, \\
& -\boldsymbol{\omega} \times \boldsymbol{e}_{3}-\lambda \boldsymbol{e}_{3} \times \boldsymbol{z}=\mathbf{0}
\end{aligned}
$$

which coincides with (4.5). As a result, proceeding as in the proof of Proposition 4.1, we show $\Omega\left(\boldsymbol{u}_{0}\right) \subseteq \mathrm{N}[\mathbf{L}]$.

We are now in a position to prove the main result of this section.

Theorem 5.3. Set $M=\max \{A, B\}$. The following properties hold. 
(i) If $C>M$ and

$$
\lambda^{2}>\frac{\beta^{2}}{C-M},
$$

then $\operatorname{Re} \sigma\left(\mathbf{L}_{1}\right) \subset(0, \infty)$.

(ii) If $C>M$ and

$$
\lambda^{2}<\frac{\beta^{2}}{C-M}
$$

then $\operatorname{Re} \sigma\left(\mathbf{L}_{1}\right) \cap(-\infty, 0) \neq \emptyset$.

(iii) If $C<M$, then $\operatorname{Re} \sigma\left(\mathbf{L}_{1}\right) \cap(-\infty, 0) \neq \emptyset$.

Proof. Our strategy in showing the theorem goes as follows. To prove $\operatorname{Re} \sigma\left(\mathbf{L}_{1}\right) \subset(0, \infty)$, it is (necessary and) sufficient to show that all solutions to (4.11) are uniformly bounded in time. To this purpose, we shall use Lemma 5.1. Conversely, to prove $\operatorname{Re} \sigma\left(\mathbf{L}_{1}\right) \cap(-\infty, 0) \neq \emptyset$, it suffices to show that there exists at least one unbounded solution, and this will be achieved by a contradiction argument that exploits Lemma 5.2. With this in mind, we now proceed to the proof of the theorem.

(i) Since $C>A, B$, Lemma 5.1 shows that solutions are bounded if (5.8) holds.

(ii) Suppose, by contradiction, $\operatorname{Re} \sigma\left(\mathbf{L}_{1}\right) \subset(0, \infty)$, then every solution to (4.10) (or, equivalently, (4.11)) must be bounded and so, by Lemma 5.2, $\Omega\left(\boldsymbol{u}_{0}\right) \subseteq \mathrm{N}[\mathbf{L}]$. By assumption and Proposition 4.1 it thus follows

$$
\Omega\left(\boldsymbol{u}_{0}\right)=\left\{\boldsymbol{v} \equiv \mathbf{0} ; \boldsymbol{\omega}=r \boldsymbol{e}_{3} ; \boldsymbol{z}=z \boldsymbol{e}_{3} ; \text { some }(r, z) \in \mathbb{R}^{2}\right\} .
$$

Thus, denoting by $\left\{t_{n}\right\}$ an unbounded sequence of times, integrating both sides of (4.9) over $\left(0, t_{n}\right)$ and taking the limit as $n \rightarrow \infty$, we obtain with $\boldsymbol{\omega}_{0}=\left(p_{0}, q_{0}, r_{0}\right)$ and $\boldsymbol{z}_{0}=\left(z_{01}, z_{02}, z_{03}\right)$

$$
0 \leq Q_{3}(r, z) \leq \mathcal{E}_{F}(\boldsymbol{v}(0))+Q_{1}\left(p_{0}, z_{01}\right)+\mathcal{Q}_{2}\left(p_{0}, z_{02}\right)+Q_{3}\left(r_{0}, z_{03}\right)
$$

where we used (5.3) and (5.4). Choosing $\boldsymbol{v}(0) \equiv \mathbf{0}, r_{0}=z_{03}=0, p_{0}=\lambda z_{01}, q_{0}=\lambda z_{02}, z_{0 i} \neq 0, i=1,2$, the previous inequality along with (5.9) entails

$$
0 \leq-\lambda^{2} z_{01}^{2}\left[A-C+\frac{\beta^{2}}{\lambda^{2}}\right]-\lambda^{2} z_{02}^{2}\left[B-C+\frac{\beta^{2}}{\lambda^{2}}\right]<0
$$

which gives a contradiction.

(iii) By contradiction, assume, instead, $\operatorname{Re} \sigma\left(\mathbf{L}_{1}\right) \subset(0, \infty)$. From $(4.11)_{4}$ it follows that $z_{3}=$ const., so that (4.9) can also be equivalently written as

$$
\frac{\mathrm{d} \mathcal{G}_{1}}{\mathrm{~d} t}+\nu\|\nabla \boldsymbol{v}\|_{2}^{2}=0
$$

where

$$
\mathcal{G}_{1}:=\|\boldsymbol{v}\|_{2}^{2}-\boldsymbol{a} \cdot \mathbb{I} \cdot \boldsymbol{a}+Q_{1}+Q_{2}+Q_{3}^{*} ; \quad Q_{3}^{*}:=C\left(\zeta_{3}-\lambda z_{3}\right)^{2} .
$$

Thus, integrating (5.12) from 0 to $t$ and arguing exactly like in (ii), we arrive again at (5.11). Now, if $C<A, B$, we get an immediate contradiction. If $A<C<B$ we take $z_{01}=0$, thus getting a contradiction again. The case $B<C<A$ is treated analogously, which complete the proof of the theorem. 


\section{Properties of the operator N}

We will now prove that the nonlinear operator $\mathbf{N}$ defined in (2.11), (2.10) satisfies the assumptions in (H4), (H5). The latter, together with the results proved in the preceding two sections, will thus allow us to use Theorem 3.2 and Theorem 3.3, and provide a complete characterization of the stability and asymptotic stability of the steady motions for (2.1); see Section 7 .

Let us use the canonical decomposition $X_{\alpha}=Y_{\alpha} \times \mathbb{R}^{3} \times \mathbb{R}^{3}$, where

$$
Y_{\alpha}:=\left\{\boldsymbol{v} \in L_{\sigma}^{2}(\mathcal{C}):\left\|\boldsymbol{A}_{0}^{\alpha} \boldsymbol{v}\right\|_{2}<\infty\right\}
$$

and the operator $\boldsymbol{A}_{0}$ is the Stokes operator introduced in (4.1). Then,

$$
Y_{\alpha} \subset H^{2 \alpha}(\mathcal{C}):=\left[L^{2}(\mathcal{C}), W^{2,2}(\mathcal{C})\right]_{\alpha}
$$

where $[\cdot, \cdot]_{\alpha}$ denotes the complex interpolation. The nonlinear operator $\mathbf{N}$ defined in (2.11), (2.10) has the bilinear structure $\mathbf{N}(\boldsymbol{u})=\boldsymbol{B}(\boldsymbol{u}, \boldsymbol{u})$ with $\boldsymbol{B}: X_{\alpha} \times X_{\alpha} \rightarrow L_{\sigma}^{2}(\mathcal{C}) \times \mathbb{R}^{3} \times \mathbb{R}^{3}$ defined by

$$
\boldsymbol{B}\left(\boldsymbol{u}_{1}, \boldsymbol{u}_{2}\right):=\boldsymbol{I}^{-1}\left(-2 \mathrm{P}\left(\boldsymbol{\omega}_{1} \times \boldsymbol{v}_{2}\right)-\mathrm{P}\left(\boldsymbol{v}_{1} \cdot \nabla \boldsymbol{v}_{2}\right),-\boldsymbol{\omega}_{1} \times \mathbb{I} \cdot\left(\boldsymbol{\omega}_{2}-\boldsymbol{a}_{2}\right),-\boldsymbol{\omega}_{1} \times \boldsymbol{z}_{2}\right)^{\top}
$$

for every $\boldsymbol{u}_{1}=\left(\boldsymbol{v}_{1}, \boldsymbol{\omega}_{1}, \boldsymbol{z}_{1}\right), \boldsymbol{u}_{2}=\left(\boldsymbol{v}_{2}, \boldsymbol{\omega}_{2}, \boldsymbol{z}_{2}\right) \in X_{\alpha}$. We recall that the operators $\boldsymbol{I}^{-1}$ and the Helmholtz projector $\mathrm{P}$ are both bounded by Lemma 3.1 and [5], Rem. III.1.1), respectively. Hence, $\mathbf{N}(\mathbf{0})=\mathbf{N}^{\prime}(\mathbf{0})=\mathbf{0}$. Moreover, since for $\mathcal{C}$ of class $C^{2}$ it is (e.g. [29], §III.2.3, Lemma III.2.4.3)

$$
\nu^{\frac{1}{2}}\|\nabla \boldsymbol{v}\|_{2}=\left\|\boldsymbol{A}_{0}^{\frac{1}{2}} \boldsymbol{v}\right\|_{2}, \quad\|\boldsymbol{v}\|_{\infty} \leq C\left\|\boldsymbol{A}_{0}^{\alpha}\right\|_{2}, \quad \boldsymbol{v} \in \mathrm{D}\left(\boldsymbol{A}_{0}\right), \alpha \in(3 / 4,1),
$$

we easily deduce, for every $\boldsymbol{v}, \boldsymbol{v}_{1}$ and $\boldsymbol{v}_{2} \in Y_{\alpha}$, and $\alpha \in(3 / 4,1)$ that

$$
\begin{aligned}
\|\mathrm{P}(\boldsymbol{v} \cdot \nabla \boldsymbol{v})\|_{2} & \leq C_{1}\left\|\boldsymbol{A}_{0}^{1 / 2} \boldsymbol{v}\right\|_{2}\left\|\boldsymbol{A}_{0}^{\alpha} \boldsymbol{v}\right\|_{2} \leq C_{2}\left\|\boldsymbol{A}_{0}^{\alpha} \boldsymbol{v}\right\|_{2}^{2} \\
\left\|\mathrm{P}\left(\boldsymbol{v}_{1} \cdot \nabla \boldsymbol{v}_{1}\right)-\mathrm{P}\left(\boldsymbol{v}_{2} \cdot \nabla \boldsymbol{v}_{2}\right)\right\|_{2} & \leq C_{3}\left[\left\|\boldsymbol{A}_{0}^{\alpha} \boldsymbol{v}_{1}\right\|_{2}\left\|\boldsymbol{A}_{0}^{1 / 2}\left(\boldsymbol{v}_{1}-\boldsymbol{v}_{2}\right)\right\|_{2}+\left\|\boldsymbol{A}_{0}^{1 / 2} \boldsymbol{v}_{2}\right\|_{2}\left\|\boldsymbol{A}_{0}^{\alpha}\left(\boldsymbol{v}_{1}-\boldsymbol{v}_{2}\right)\right\|_{2}\right] \\
& \leq C_{4}\left[\left\|\boldsymbol{A}_{0}^{\alpha} \boldsymbol{v}_{1}\right\|_{2}+\left\|\boldsymbol{A}_{0}^{\alpha} \boldsymbol{v}_{2}\right\|_{2}\right]\left\|\boldsymbol{A}_{0}^{\alpha}\left(\boldsymbol{v}_{1}-\boldsymbol{v}_{2}\right)\right\|_{2}
\end{aligned}
$$

This shows that the remaining conditions in (H4) are satisfied with $p=2$ and $\alpha$ as above. We next pass to the proof of (H5). To this end, let

$$
\boldsymbol{u}=\boldsymbol{u}^{(0)}+\boldsymbol{u}^{(1)}, \quad \boldsymbol{u}^{(0)}=\left(\boldsymbol{v} \equiv \mathbf{0}, \boldsymbol{\omega}^{(0)}, \boldsymbol{z}^{(0)}\right) \in \mathrm{N}(\mathbf{L}), \boldsymbol{u}^{(1)}=\left(\boldsymbol{v}, \boldsymbol{\omega}^{(1)}, \boldsymbol{z}^{(1)}\right) \in \mathrm{R}(\mathbf{L}) .
$$

Then, from (2.10) we deduce

$$
\begin{aligned}
\mathbf{M}\left(\boldsymbol{u}^{(0)}, \boldsymbol{u}^{(1)}\right)=\left(-2 \mathrm{P}\left[\left(\boldsymbol{\omega}^{(0)}+\boldsymbol{\omega}^{(1)}\right) \times \boldsymbol{v}\right]-\mathrm{P}(\boldsymbol{v} \cdot \nabla \boldsymbol{v}),\right. & \\
& \left.-\left(\boldsymbol{\omega}^{(0)}+\boldsymbol{\omega}^{(1)}\right) \times \mathbb{I} \cdot\left(\boldsymbol{\omega}^{(0)}+\boldsymbol{\omega}^{(1)}-\boldsymbol{a}\right),-\left(\boldsymbol{\omega}^{(0)}+\boldsymbol{\omega}^{(1)}\right) \times\left(\boldsymbol{z}^{(0)}+\boldsymbol{z}^{(1)}\right)\right)^{\top} .
\end{aligned}
$$

Thus, if $K_{A}, K_{B} \neq 1$, from Proposition 4.1 we infer

$$
\boldsymbol{\omega}^{(0)} \times \mathbb{I} \cdot \boldsymbol{\omega}^{(0)}=\boldsymbol{\omega}^{(0)} \times \boldsymbol{z}^{(0)}=\mathbf{0},
$$


and (H5) follows from the latter, (6.4) and (6.3).

\section{Nonlinear Stability PROperties}

In view of the results obtained in the previous three sections, we are now able to employ the general theory developed in Section 3, and use Theorem 3.2 and Theorem 3.3 to provide a complete characterization of the stability and asymptotic stability properties of the steady-state motion (2.4).

We begin to show the following nonlinear stability result.

Theorem 7.1. (Nonlinear stability) Let $\alpha \in(3 / 4,1)$ and assume that the following conditions hold

$$
C>M \equiv \max \{A, B\} ; \quad \lambda^{2}>\frac{\beta^{2}}{C-M} .
$$

Then, the steady-state (2.4) is stable in $X_{\alpha}$, namely, for any $\varepsilon>0$ there is $\delta>0$ such that

$$
\left\|\left(\boldsymbol{v}_{0}, \boldsymbol{\omega}_{0}, \boldsymbol{z}_{0}\right)\right\|_{\alpha}<\delta \Longrightarrow \sup _{t \geq 0}\|(\boldsymbol{v}(t), \boldsymbol{\omega}(t), \boldsymbol{z}(t))\|_{\alpha}<\varepsilon .
$$

Moreover, there exists $r \in \mathbb{R}$ such that

$$
\begin{aligned}
& \lim _{t \rightarrow \infty}\left\|\boldsymbol{A}_{0}^{\alpha} \boldsymbol{v}(t)\right\|_{2}=0, \\
& \lim _{t \rightarrow \infty} \boldsymbol{\omega}(t)=r \boldsymbol{e}_{3} \\
& \lim _{t \rightarrow \infty} \boldsymbol{z}(t)=\mathbf{0}
\end{aligned}
$$

all the above limits occurring at an exponential rate.

Proof. By Theorem 5.3, we know that, under the stated assumptions (7.1), we have $\sigma\left(\mathbf{L}_{1}\right) \subset(0, \infty)$. Therefore, in view of what we have shown in Proposition 4.1-Proposition 4.4, and Sections 5 and $6,{ }^{3}$ we may employ Theorem 3.2 and deduce (7.2) and (7.3) 1,2 . Furthermore, there exists $z \in \mathbb{R}$ such that

$$
\lim _{t \rightarrow \infty} \boldsymbol{z}(t)=z \boldsymbol{e}_{3}
$$

at exponential rate. We now observe that the perturbation field $\boldsymbol{z}(t)$ must satisfy (2.7) (or, equivalently, the data satisfy (2.8)). This implies that either $z=2$ or else $z=0$. In the first case, taking into account (2.5), we deduce that the terminal state of the coupled system will be of the type $\left(\boldsymbol{v} \equiv \mathbf{0}, \boldsymbol{\omega}=r \boldsymbol{\gamma}, \boldsymbol{\gamma}=\boldsymbol{e}_{3}\right)$ which, as it is easily checked, is a steady-state solution to (2.1) corresponding to a constant rigid rotation of $\mathcal{S}$ around $\boldsymbol{e}_{3}$ with its center of mass $G$ in its lowest position. However, by the stability property (7.1), $G$ must be at all times in a neighborhood of its highest position. Therefore, we can only have $z=0$, which completes the proof of the theorem.

Remark 7.2. As an illustration of the results obtained in the previous theorem, consider a "classical" symmetric top, $\mathcal{T}$, spinning at sufficiently fast rate around its axis a in the vertical direction, $d$, passing through the fixed point $O$ and center of mass $G$ (in its highest position). It is then well known (e.g. [22], Ex. 9.7C) that a small disorientation of a from $d$ will produce a stable precession of $\mathcal{T}$ around $d$ with a performing small oscillations (nutation). If, however, $\mathcal{T}$ possess an interior cavity filled up with a viscous liquid, Theorem 7.1 tells us that under the same above circumstances, the axis a will eventually reposition itself in the vertical direction $d$, at an exponential rate. This fact provides a further example of the stabilizing influence of an interior liquid-filled cavity on the motion of a rigid body $[4,8,10,17-20]$.

\footnotetext{
${ }^{3}$ Notice that, in view of $(7.1), K_{A}, K_{B} \neq 1$.
} 
We also have the following instability result, which is an immediate consequence of Proposition 4.1, Proposition 4.2, Theorem 5.3, Section 6, and Theorem 3.3.

Theorem 7.3 (Nonlinear instability). With the same notation as in Theorem 7.1, the steady state (2.4) is unstable in $X_{\alpha}$ if either

$$
C>M, \text { and } \lambda^{2}<\frac{\beta^{2}}{C-M}
$$

or

$$
C<M \text {. }
$$

\section{REFERENCES}

[1] V.V. Chernousko, Motion of a Rigid Body with Cavities Containing a Viscous Fluid, NASA Technical Translations, Moscow (1972).

[2] F.L. Chernousko, L.D. Akulenko, and D.D. Leshchenko, Evolution of motions of a rigid body about its center of mass. Springer, Cham (2017).

[3] J.L. Daleckii and M.G. Krein, Stability of solutions of differential equations in Banach spaces. Vol. 43 of Translations of Mathematical Monographs. AMS, R.I. Providence (1974).

[4] K. Disser, G.P. Galdi, G. Mazzone and P. Zunino, Inertial motions of a rigid body with a cavity filled with a viscous liquid. Arch. Ration. Mech. Anal. 221 (2016) 487-526.

[5] G.P. Galdi, An introduction to the mathematical theory of the Navier-Stokes equations. Steady-state problems, Second edition. Springer Monographs in Mathematics. Springer, New York (2011)

[6] G.P. Galdi, Stability of permanent rotations and long-time behavior of inertial motions of a rigid body with an interior liquid-filled cavity. Ch. in Particles in flows. Advances in Mathematical Fluid Mechanics. Springer (2017) $217-253$.

[7] G.P. Galdi and G. Mazzone, On the motion of a pendulum with a cavity Entirelyn filled with a viscous liquid. Ch in Recent progress in the theory of the Euler and Navier-Stokes Equations. London Mathematical Society Lecture Note Series: 430. Cambridge University Press (2016) 37-56.

[8] G.P. Galdi and G. Mazzone, Stability and long-time behavior of a pendulum with an interior cavity filled with a viscous liquid. Mathematical Analysis of Viscous Incompressible Fluid. RIMS Kôkyûroku Proceedings No. 2058. (2018) 90-107.

[9] G.P. Galdi, G. Mazzone and M. Mohebbi, On the motion of a liquid-filled rigid body subject to a time-periodic torque. Recent developments of mathematical fluid mechanics. In: Advances in Mathematical Fluid Mechanics. Springer Basel (2016) $233-255$.

[10] G.P. Galdi, G. Mazzone and M. Mohebbi, On the motion of a liquid-filled heavy body around a fixed point. Quart. Appl. Math. 76 (2018) 113-145.

[11] I. Gohberg and S. Goldberg, Classes of linear operators, Vol. 1. Birkhäuser-Verlag, Basel-Boston-Berlin (1990)

[12] D. Henry, Geometric theory of semilinear parabolic equations. In Vol. 840 of Lecture Notes in Mathematics. Springer-Verlag, Berlin-New York (1981)

[13] T. Kato, Perturbation Theory for Linear Operators. Springer-Verlag, Berlin-New York (1966)

[14] N.D. Kopachevsky and S.G. Krein, Operator Approach to Linear Problems of Hydrodynamics, Vol.2: Nonself-Adjoint Problems for Viscous Fluids. Birkhäuser Verlag, Basel-Boston-Berlin (2000).

[15] A.G.A. Kostyuchenko, A. Shkalikov and Y.M. Yu, On the stability of a top with a cavity filled with viscous fluid (Russian). Funktsional. Anal. i Prilozhen. 32 (1998) 36-55; English transl., Funct. Anal. Appl. 32 (1998) 100-113.

[16] A. Lunardi, Analytic semigroups and optimal regularity in parabolic problems. In Vol. 16 of Progress in Nonlinear Differential Equations and their Applications. Birkhäuser Verlag, Basel (1995).

[17] G. Mazzone, A mathematical analysis of the motion of a rigid body with a cavity containing a newtonian fluid. Ph.D. thesis, Università del Salento (2012).

[18] G. Mazzone, On the dynamics of a rigid body with cavities completely filled by a viscous liquid. Ph.D. thesis, University of Pittsburgh (2016).

[19] G. Mazzone, J. Prüss and G. Simonett, On the motion of a fluid-filled rigid body with Navier boundary conditions. SIAM J. Math. Anal. 51 (2019) 1582-1606.

[20] G. Mazzone, J. Prüss and G. Simonett, A maximal regularity approach to the study of motion of a rigid body with a fluid-filled cavity. J. Math. Fluid Mech. 21 (2019) 44.

[21] N.N. Moiseyev and V.V. Rumyantsev, Dynamic stability of bodies containing fluid. Springer, New York (1968).

[22] L.A. Pars, A treatise on analytical dynamics. John Wiley \& Sons, Inc., New York (1965).

[23] A. Pazy, Semigroups of linear operators and applications to partial differential equations. In Vol. 44 of Applied Mathematical Sciences. Springer-Verlag, New York (1983).

[24] J. Prüss, G. Simonett and R. Zacher, On convergence of solutions to equilibria for quasilinear parabolic problems. J. Differ. Equ. 246 (2009) 3902-3931. 
[25] V.V. Rumiantsev, On the stability of stationary motions of rigid bodies with cavities containing fluid. Prikl. Mat. Meh. 26 977-991 (Russian); translated as J. Appl. Math. Mech. 26 (1963) 1485-1505.

[26] E.P. Smirnova, Stabilization of free rotation of an asymmetric top with cavities completely filled with a liquid. Prikl. Mat. Meh. 38 (1974) 980-985.

[27] E.P. Smirnova, Motion of a high vuscous liquid in a rotating torus. Prikl. Mat. Meh. 39 (1975) 177-182.

[28] S.L. Sobolev, On a new problem of mathematical physics (Russian). Izv. Akad. Nauk SSSR. Ser. Mat. 18 (1954) 3-50.

[29] H. Sohr, The Navier-Stokes Equations, An Elementary Functional Analytic Approach. Birkhäuser Verlag, Basel (2001).

[30] A.E. Taylor, Introduction to functional analysis. John Wiley \& Sons, Inc., New York; Chapman \& Hall, Ltd., London (1958).

[31] M. Yu Yurkin, On the stability of small oscillations of a rotating asymmetric top with fluid inside. Dokl. Akad. Nauk 362 (1998) 170-173. 